3. Флетчер К. Численные методы на основе метода Галеркина. М. : Мир, 1988. 352 с.

4. Коул Дж. Методы возмущений в прикладной математике. М. : Мир, 1972. 274 с.
5. Андрейченко Д. К., Андрейченко К. П. К теории комбинированных динамических систем // Изв. РАН. Теория и системы управления. 2000. № 3. С. 54-69.

\title{
On Stability Theory of Autonomous Angular Stabilization System for Combined Dynamical Systems
}

\author{
D. K. Andreichenko ${ }^{1}$, K. P. Andreichenko ${ }^{2}$, V. V. Kononov ${ }^{1}$ \\ ${ }^{1}$ Saratov State University, Russia, 410012, Saratov, Astrahanskaya st., 83, AndreichenkoDK@info.sgu.ru, \\ valentin.kononov@gmail.com \\ ${ }^{2}$ Saratov State Technical University, Russia, 410054, Saratov, Polytechnicheskaya st., 77, kp_andreichenko@ @enet.ru \\ Studied the effect on the stability of the longitudinal acceleration discretely-continuum model of single-channel angular stabilization \\ system with of delayed argument. Methods of construction asymptotic stability areas and analysis of impulse transition functions are \\ developed. The critical values of the longitudinal acceleration are defined.
}

Key words: combined dynamical systems, stabilization systems.

\section{References}

1. Andreichenko D. K., Andreichenko K. P. On the theory of autonomous angular stabilization systems of missiles for salvo firing. J. of Computer and Systems Sciences Intern., 2009, vol. 48, no. 3, pp. 465-480. DOI: 10.1134/S1064230709030137.

2. Andreichenko D. K., Andreichenko K. P. On the theory of stabilization of satellites having elastic rods. J. of Computer and Systems Sciences Intern., 2004, vol. 43, no. 6, pp. 973-986.

3. Fletcher K. Chislennye metody na osnove metoda
Galjorkina [Numerical methods based on the Galerkin method]. Moscow, Mir, 1988, 352 p.

4. Cole J. D. Perturbation methods in applied mathematics. Blaisdell Publishing Co. Ginn and Co., Waltham, Mass.-Toronto, Ont.-London, 1968, 260 p. (Rus. ed.: Cole J. Metody vozmushhenij v prikladnoj matematike. Moscow, Mir, 1972, 274 p.)

5. Andreichenko D. K., Andreichenko K. P. On the theory of hybrid dynamical systems. J. of Computer and Systems Sciences Intern., 2000, vol. 39, no. 3, pp. 383-398.

\section{УДК 519.95}

\section{ИДЕНТИФИКАЦИЯ СТРУКТУРЫ АВТОМАТА ФРАГМЕНТАМИ ПОВЕДЕНИЯ}

\section{С. А. Богомолов}

Кандидат физико-математических наук, доцент касредры прикладной математики и инфрорматики, Саратовский государственный социально-экономический университет, Alexbogomolov@ya.ru

\begin{abstract}
Изучается задача идентификации структуры автомата конечным фррагментом его поведения. Под поведением автомата понимается множество различных о.-д. фрункций, реализуемых в автомате, а под конечным сррагментом поведения -следы о.-д. фуункцй и автоматов. Ведено понятие идентифрицирующего следа для автомата, «неизбыточного» относительно его реализации. Предложен подход, позволяющий в множестве следов, идентисицирующих автомат, выделить и описать конечное множество «неизбыточных» следов, содержащих только необходимую инсрормацию для идентификации автомата.
\end{abstract}

Ключевые слова: автомат, эксперимент с автоматом, подэксперимент эксперимента, след о.-д. фуунции и автомата, идентисрицирующий след автомата, операция редукции следа, неизбыточный идентифицииующий след автомата.

\section{ВВЕДЕНИЕ}

Обширный класс как фундаментальных, так и прикладных проблем анализа дискретных систем допускает их естественное сведение к проблемам идентификации. Л. Льюинг [1] отмечал: «Формирование моделей на основе результатов наблюдений и исследование их свойств - вот по существу основное содержание науки». Модели могут быть более или менее формализованными, но все они 
обладают главной особенностью, что связывает наблюдения в некоторую общую картину. Решение задачи построения математических моделей динамических систем по данным наблюдений их поведения составляют предмет теории идентификации. Таким образом, идентификация - это один из основных способов формирования математических моделей, в котором используется регистрация экспериментальных данных, представленных в виде входных и выходных сигналов, и модель формируется в результате обработки соответствующих данных. В настоящей работе термин идентификация используется только применительно к уровню математических моделей, для которых в качестве модельной структуры выбрана модель конечного автомата. Тогда под идентификацией понимают процедуру построения оптимальной, в определенном смысле, структуры дискретного автомата по результатам подачи входных воздействий и наблюдения соответствующих выходных реакций.

В работах $[2,3]$ предложен конструктивный подход, в рамках которого в множестве автоматов, реализующих заданный конечный фрагмент вход-выходного поведения, выделено конечное множество «неизбыточных» автоматов, содержащих только необходимую информацию для реализации данного поведения. Основной результат состоит в решении задачи анализа, а именно - описание свойств и структуры конечных фрагментов поведения (следов автоматов), идентифицирующих автомат как «неизбыточный» - неприводимый, относительно их реализации. Анализ полученных результатов $[2,3]$ показывает, что при исследовании проблемы идентификации структуры автоматов необходимо изучение следующих вопросов.

1. Какова предельная (граничная) информация, при которой указанное идентифицирующее поведение того или иного вида существует и без которой идентификация невозможна.

2. Какова структура указанного описания поведения и свойств автомата, что обязательно должно присутствовать в описании, а что является издержками алгоритма получения описаний.

Эти вопросы являются не только актуальными, но и их решение определяет эффективность того или иного способа задания конечных автоматов с функциональной точки зрения как абстрактной модели алгоритмов переработки дискретной информации.

\section{1. ОПРЕДЕЛЕНИЯ И ПОНЯТИЯ, НЕОХОДИМЫЕ ДЛЯ ИЗЛОЖЕНИЯ МАТЕРИАЛА}

Под автоматом понимается конечный полностью определенный детерминированный автомат Мили [4] с фиксированными конечными входным и выходным алфавитами. Пусть $A=(S, X, Y, \delta, \lambda)-$ автомат, у которого $S=\left\{s_{1}, s_{2}, \ldots, s_{n}\right\}, X=\left\{x_{1}, x_{2}, \ldots, x_{m}\right\}, Y=\left\{y_{1}, y_{2}, \ldots, y_{p}\right\}-$ конечные множества состояний, входной и выходной алфавиты соответственно; $\delta$ и $\lambda-$ функции переходов и выходов, отображающие множество $S \times X$ в $S$ и $Y$. Обозначим через $X^{*}\left(Y^{*}\right)$ множество всех непустых слов конечной длины в алфавите $X(Y)$. Функции $\delta$ и $\lambda$ естественным образом [4] распространяются на множество $S \times X^{*}$ со значениями в $S$ и $Y^{*}$ соответственно. Множество всех автоматов с фиксированными входным $X$ и выходным $Y$ алфавитами обозначим через $K(U)$, где $U=(X \times Y)$. Обозначим через $U^{*}$ множество всех пар слов $(\alpha, \beta)$ таких, что $\alpha \in X^{*}, \beta \in Y^{*}$ и длины слов $\alpha$ и $\beta$ совпадают. Пару слов одинаковой длины $(\alpha, \beta)=\left(x_{1} \ldots x_{r}, y_{1} \ldots y_{r}\right)$ назовем вход-выходным словом, допустимым автоматом $A$ в состоянии $s$, если $\lambda\left(s, x_{1} \ldots x_{r}\right)=y_{1} \ldots y_{r}$. Множество вход-выходных слов, допустимых в состоянии $s$ автоматом $A$, очевидно, является функциональным отношением, которое обозначим через $\varphi_{s}$ и назовем автоматным отображением, реализуемым состоянием $s$ автомата $A$. Конечное множество вход-выходных слов $R \subset U^{*}$ назовем экспериментом, допустимым в состоянии $s \in S_{A}$ автоматом $A$, если всякое слово из $R$ есть вход-выходное слово, допустимое в состоянии $s$ автоматом $A$. Если $R$ содержит единственное слово из $U^{*}$, то $R$ - простой эксперимент, в противном случае $R$ - кратный эксперимент. Множество $R(\alpha, \beta)$ всех тех и только тех слов $\left(\alpha^{\prime}, \beta^{\prime}\right)$, для которых $(\alpha, \beta)\left(\alpha^{\prime}, \beta^{\prime}\right) \in R$, назовем подэкспериментом эксперимента $R$ по слову $(\alpha, \beta)$ [3]. Обозначим через $[R]$ множество всех собственных префиксов вход-выходных слов эксперимента $R$, а через $B(R)$ множество всех непустых подэкспериментов эксперимента $R$ по словам из $[R]$, тогда $B(W)=\bigcup_{R \in W} B(R)$.

Эксперименты $R_{1}$ и $R_{2}$ назовем совместимыми, если для любых $\left(\alpha_{1}, \beta_{1}\right)$ и $\left(\alpha_{2}, \beta_{2}\right)$, являющихся префиксами некоторых вход-выходных слов из $R_{1}$ и $R_{2}$ соответственно, из равенства $\alpha_{1}=\alpha_{2}$ следует, что $\beta_{1}=\beta_{2}$.

В качестве поведения [4] автомата будем рассматривать множество всех попарно различных 
ограниченно-детерминированных функций (о.-д. функций), в нем реализуемых. Через $\Omega(U)$ обозначим множество всех о.-д. функций, отображающих $X^{*}$ в $Y^{*}$. Пусть $f-$ о.-д. функция из $\Omega(U)$. Произвольное множество $R \in U^{*}$ назовем следом о.-д. функции $f$, если для любого $(\alpha, \beta) \in R$ имеет место $f(\alpha)=\beta$. След $R$ о.-д. функции $f$ назовем собственным, если существует $(\alpha, \beta) \in U^{*}$ такое, что $f(\alpha)=\beta$, но $(\alpha, \beta) \notin R$. След $R$ о.-д. функции $f$ назовем конечным, если $R$ содержит конечное множество слов из $U^{*}$. При изложении материала под следом будем понимать только конечный след. След $R$ о.-д. функции $f-$ простой, если он содержит единственное слово из $U^{*}$, и кратный - в противном случае. Пусть $F=\left\{f_{1}, \ldots, f_{n}\right\}-$ конечная система о.-д. функций из $\Omega(U)$ и $W=\left(R_{1}, \ldots, R_{k}\right)-$ конечная совокупность конечных подмножеств из $U^{*}$. Множество $W=\left(R_{1}, \ldots, R_{k}\right)$ назовем следом системы о.-д. функций $F=\left\{f_{1}, \ldots, f_{n}\right\}$, если для любого $i \in\{1, \ldots, n\}$ существует $j \in\{1, \ldots, n\}$ такое, что $R_{i}$ есть след о.-д. функции $f_{j}$.

Пусть $A \in K(U)$. Через $F(A)=\left\{f_{1}, \ldots, f_{n}\right\}$ обозначим множество всех попарно различных о.-д. функций, реализуемых в автомате $A$, образующих полное поведение автомата. Следом $W=\left(R_{1}, \ldots, R_{k}\right)$ автомата $A$ назовем произвольный конечный след системы $F(A)$. След $W=\left(R_{1}, \ldots, R_{n}\right)$ автомата $A-$ простой, если для любого $j \in\{1, \ldots, n\} R_{i}$ содержит единственное слово из $U^{*}$. Простой след автомата представляет конечное множество вход-выходных слов, допустимых (возможно, различными состояниями) автомата, полученных в результате проведения экспериментов или же априорно заданных в качестве условий функционирования синтезируемого автомата. След автомата $W=\left(R_{1}, \ldots, R_{n}\right)$ назовем кратным (структурированным), если существует $j \in\{1, \ldots, n\}$ такое, что $R_{j}$ есть кратный след некоторой о.-д. функции из $F(A)$. Кратные следы возникают при введении дополнительной информации для множества вход-выходных слов, реализуемых идентифицируемым автоматом, а именно указанием о допустимости некоторыми состояниями автомата определенных подмножеств из исходного множества вход-выходных слов. Таким образом, след автомата представляет собой конечное множество экспериментов конечной длины, допустимых с данным автоматом. В работе [2] описаны структура и свойства следов автомата, идентифицирующих заданный автомат как неприводимый относительно реализации указанных следов.

В настоящей работе рассмотрены вопросы оптимизации структуры и сложности следов автомата, идентифицирующих его как неприводимый (относительно реализации), а именно поиску и описанию свойств «неизбыточных» следов автомата, содержащих лишь информацию, необходимую для идентификации и наиболее простых по структуре.

\section{2. ОПТИМИЗАЦИЯ ИДЕНТИФИЦИРУЮЩИХ СЛЕДОВ АВТОМАТА}

Пусть $A-$ приведенный автомат из $K(U)$. Через $C(A)=\left\{W_{A}\right\}$ обозначим множество следов автомата $A$, идентифицирующих автомат $A$ как неприводимый в классе $K\left(W_{A}\right)$. Элементы множества $C(A)$ представляют собой конечное множество кратных экспериментов $W=\left(R_{1}, \ldots, R_{k}\right)$, допустимых в автомате $A$ таких, что $A$ является единственным неприводимым автоматом в классе $K(W)$. В дальнейшем под $B(W)$ будем понимать множество всех попарно различных подэкспериментов экспериментов из $W$, включая и эксперименты $R_{1}, \ldots, R_{k}$. На множестве следов $C(A)$ рассмотрим следующие отношения.

Пусть $R_{1}, R_{2}$ - эксперименты, допустимые некоторым автоматом из $K(U)$, тогда $R_{1} \supseteq R_{2}$, если любое вход-выходное слово из $R_{1}$ принадлежит множеству $R_{2}$. Эксперимент $R_{2}$ покрывает $R_{1}$ $\left(R_{1} \leq R_{2}\right)$, если существует эксперимент $R^{\prime} \in B\left(R_{2}\right)$ такой, что любое вход-выходное слово из $R_{1}$ является префиксом (возможно, несобственным) некоторого вход-выходного слова из $R^{\prime}$. Пусть $W_{1}, W_{2}$ - следы из $C(A)$. След $W_{2}$ покрывает след $W_{1}\left(W_{1} \leq W_{2}\right)$, если для любого эксперимента $R \in W_{1}$ существует эксперимент $R^{\prime} \in B\left(W_{1}\right)$ такой, что $R \leq R^{\prime}$. Отношение покрытия « $\leq$ на множестве следов $C(A)$ удовлетворяет условиям транзитивности и рефлексивности, но в общем случае не является антисимметричным и, таким образом, определяет в $C(A)$ некоторый частичный предпорядок. След $W=\left(R_{1}, \ldots, R_{k}\right)$ из $C(A)$ назовем приведенным, если для любого $i \in\{1, \ldots, k\}$ эксперимент $R_{i}$ не покрывается никаким экспериментом $R_{j} \in W$, если $i \neq j, j \in\{1, \ldots, k\}$. Множество всех приведенных следов из $C(A)$ бесконечно и содержит, вообще говоря, следы с «избыточной» информацией относительно однозначного задания автомата $A$. Это обусловлено следующим: с каждым следом $W$ из $C(A)$ множество $C(A)$ содержит след $W U W^{\prime}$, где $W^{\prime}-$ множество экспериментов, допустимых 
в $A$; бесконечное множество систем экспериментов, покрывающих $W$, так как если $W \in C(A)-$ множество экспериментов, допустимых в $A$, то $W^{\prime} \in C(A)$. След $W \in C(A)$ назовем тупиковым в $C(A)$, если для любого следа $W^{\prime} \in C(A)$ такого, что $W \geq W^{\prime}$, справедливо $W=W^{\prime}$.

Приведем основные свойства тупиковых следов из $C(A): 1)$ тупиковые следы множества $C(A)$ не покрывают никакие следы из $C(A)$; из теоремы 2 [3] следует; 2) что любой тупиковый след множества $C(A)$ содержит $n$ экспериментов, если $A$ имеет $n$ состояний; 3$)$ если $W=\left(R_{1}, \ldots, R_{n}\right)-$ тупиковый из $C(A)$, где $\left|S_{A}\right|=n$, то для любого $i \in\{1, \ldots, n\}-$ эксперимент $R_{i}$ допустим в $A$ состоянием $s_{i} \in S_{A}$, но не допустим в $A$ никаким состоянием $s_{j} \in S_{A}, j \in\{1, \ldots, n\}$, отличным от $s_{i}$; для всякого $x \in X$ и некоторого $y \in Y$ существует $j \in\{1, \ldots, n\}$, зависящее от $x$, такое, что подэксперимент $R_{i}(x, y)$ совместим с $R_{j}$ и не совместим с $R_{1}, \ldots, R_{j-1}, R_{j+1}, R_{n}$.

Утверждение. Для любого вход-выходного алфавита $U=X \times Y,|X| \geq 2,|Y| \geq 2$ множество $K(U)$ содержит автоматы $A_{1}$ и $A_{2}$, такие, ито множество тупиковых следов: а) в $C\left(A_{1}\right)-$ счетное; б) в $C\left(A_{2}\right)$ - конечное.

Доказательство. Не ограничивая общности, полагаем, что $X=Y=\{0,1\}$.

Рассмотрим автомат $A_{1}=\left\{S_{1}=\left\{s_{1}^{1}, s_{2}^{1}\right\},\{0,1\},\{0,1\}, \delta_{1}, \lambda_{1}\right\}$,

$$
\begin{aligned}
& \delta_{1}\left(s_{1}^{1}, 0\right)=s_{1}^{1}, \quad \delta_{1}\left(s_{1}^{1}, 1\right)=s_{1}^{1}, \quad \delta\left(s_{2}^{1}, 0\right)=s_{2}^{1}, \quad \delta\left(s_{2}^{1}, 1\right)=s_{2}^{1}, \\
& \lambda\left(s_{1}^{1}, 0\right)=0, \quad \lambda\left(s_{1}^{1}, 1\right)=1, \quad \lambda\left(s_{2}^{1}, 0\right)=0, \quad \lambda\left(s_{2}^{1}, 1\right)=0 .
\end{aligned}
$$

Рассмотрим множество следов $W^{(n)}=\left\{R_{1}^{(n)}, R_{2}^{(n)}\right\}$ автомата $A_{1}$, где $R_{1}^{(n)}$ и $R_{1}^{(n)}$ определяются следующим образом:

$$
R_{1}^{(n)}=\left\{(0,0)^{n-1}(1,1) ;(1,1)(0,0)^{n}(1,1)\right\}, \quad R_{2}^{(n)}=\left\{(1,0)(1,0) ;(0,0)^{n}(1,0) ;(0,0)^{n-2}(1,0)\right\},
$$

где $(x, y)^{k}-$ слово, содержащее $k$ символов $(x, y) \in U$. Любой след данного множества для любого $n \geq 3$ удовлетворяет условию теоремы 2 [3] и, следовательно, $W^{(n)} \in C\left(A_{1}\right)$. Кроме того, для любого $n \geq 3$ след $W^{(n)}$ - тупиковый элемент из $C\left(A_{1}\right)$ и, следовательно, в $C\left(A_{1}\right)$ содержится счетное множество тупиковых следов автомата $A_{1}$.

Рассмотрим автомат $A_{2}=\left\{S_{1}=\left\{s_{1}^{1}, s_{2}^{1}\right\},\{0,1\},\{0,1\}, \delta_{2}, \lambda_{2}\right\}$,

$$
\begin{array}{ccc}
\delta_{2}\left(s_{1}^{2}, 0\right)=s_{1}^{2}, & \delta_{2}\left(s_{1}^{2}, 1\right)=s_{2}^{2}, & \delta_{2}\left(s_{2}^{2}, 0\right)=s_{2}^{2}, \\
\lambda\left(s_{1}^{2}, 0\right)=0, \quad \lambda\left(s_{1}^{2}, 1\right)=1, & \left.\delta_{2}\left(s_{2}^{2}, 1\right)=s_{1}^{2}, 0\right)=1, & \lambda\left(s_{2}^{1}, 1\right)=0 .
\end{array}
$$

Из второго свойства тупиковых следов множества $C\left(A_{2}\right)$ содержат ровно два эксперимента. Пусть $W_{2}=\left(R_{1}, R_{2}\right)$ - произвольный тупиковый элемент множества $C\left(A_{2}\right)$. Рассмотрим подэксперименты $R_{i}(x, y)$, где $i \in\{1,2\}, x \in X, y \in Y$. Для того чтобы подэксперимент $R_{i}(x, y)$ эксперимента $R_{i}$ из $W_{2}$ удовлетворял третьему свойству тупиковых элементов $C\left(A_{2}\right)$ и теореме 2 из [3], достаточно, чтобы этот подэксперимент был не пуст и содержал любой символ $\left(x^{\prime}, y^{\prime}\right) \in U$ такой, что $(x, y)\left(x^{\prime}, y^{\prime}\right) \in R_{i}$. Тогда любой эксперимент $R_{i}^{\prime}$, полученный в результате «продолжения» эксперимента $R_{i}$ длины $r \geq 3$, будет покрывать след $W_{2}$, в котором для любых $i \in\{1,2\}, x \in X$ и некоторого $y \in Y$ подэксперимент $R_{i}(x, y)$ есть буква алфавита $U$. Следовательно, длина всех вход-выходных слов следа $W_{2}$ равна двум, а число экспериментов, имеющих фиксированную длину и конечный вход-выходной алфавит $U$, конечно, что означает конечность тупиковых следов множества $C\left(A_{2}\right)$. Утверждение доказано.

Из этого утверждения следует, что существуют автоматы, множества тупиковых следов, их идентифицирующих, содержат «избыточную» информацию относительно идентификации автомата в рамках выбранного подхода. В этой связи предполагается рассматривать «неизбыточные» тупиковые следы автомата, идентифицирующие его с использованием некоторого преобразования экспериментов операции редукции следа.

Пусть $R=\{\alpha, \beta\}-$ простой эксперимент, допустимый некоторым автоматом из $K(U)$ длины не менее трех, а $\alpha_{1}, \beta_{1}, \alpha_{2}, \beta_{2}$ - префиксы (возможно, несобственные) слова $\alpha, \beta$, причем $\alpha_{1}, \beta_{1}-$ префикс $\alpha_{2}, \beta_{2}$. Таким образом, эксперимент $R$ можно представить в виде $R=\left(\alpha_{1}, \beta_{1}\right) R\left(\alpha_{2}, \beta_{2}\right)=$ $=\left(\alpha_{2}, \beta_{2}\right) R\left(\alpha_{2}, \beta_{2}\right)$.

Определение 1. Полагаем, что эксперимент $R^{\prime}\left(\alpha_{1}, \beta_{1}\right) R\left(\alpha_{2}, \beta_{2}\right)$ получен из эксперимента $R$ путем применения операции редукции $\tau$ (сокращения) эксперимента: $\tau: R^{\prime}\left(\alpha_{1}, \beta_{1}\right) \rightarrow R\left(\alpha_{2}, \beta_{2}\right)$, если 
в эксперименте $R$ подэксперимент $R\left(\alpha_{1}, \beta_{1}\right)$ заменен на подэксперимент $R\left(\alpha_{2}, \beta_{2}\right)$, подэксперимент эксперимента $R\left(\alpha_{1}, \beta_{1}\right)$.

Заметим, что эксперимент, полученный в результате применения операции сокращения в общем случае, может не допускаться автоматом, допускающим эксперимент $R$, к которому эта операция была применима. Префиксы $\left(\alpha_{1}, \beta_{1}\right),\left(\alpha_{2}, \beta_{2}\right)$ эксперимента $R$, определяющие заменяемый и замещающий подэксперименты назовем параметрами операции редукции эксперимента. В зависимости от выбора параметров при применении операции редукции следа к простому эксперименту $R$ возможны следующие ситуации:

1. Пусть $\left(\alpha_{1}, \beta_{1}\right)=l_{U}$, где $l_{U}-$ пустое слово в $U^{*}$. Тогда $\left(\alpha_{2}, \beta_{2}\right)=(\alpha, \beta)$ и $\tau(R)=l_{U}$, так как $R\left(\alpha_{1}, \beta_{1}\right)=(\alpha, \beta), R\left(\alpha_{2}, \beta_{2}\right)=(\alpha, \beta)$.

2. Пусть $\left(\alpha_{1}, \beta_{1}\right)=\left(\alpha_{2}, \beta_{2}\right) \neq l_{U}$. Тогда $\tau(R)=R-$ тождественное (тривиальное) преобразование.

3. Пусть $\left(\alpha_{1}, \beta_{1}\right) \neq l_{U}$ и является собственным префиксом $(\alpha, \beta)$, а $\left(\alpha_{2}, \beta_{2}\right)=(\alpha, \beta)$. Тогда $\left(\alpha_{2}, \beta_{2}\right)=l_{U}$ и $(R)=\left(\alpha_{1}, \beta_{1}\right)$, так как $R\left(\alpha_{1}, \beta_{1}\right)$ заменяется на $l_{U}$.

4. Пусть $\left(\alpha_{1}, \beta_{1}\right)=l_{U},\left(\alpha_{2}, \beta_{2}\right) \neq l_{U}-$ собственный префикс $(\alpha, \beta)$. Тогда $R\left(\alpha_{1}, \beta_{1}\right)=(\alpha, \beta)$ и $\tau(R)=R\left(\alpha_{2}, \beta_{2}\right)-$ суффикс слова $(\alpha, \beta)$.

5. Пусть $\left(\alpha_{1}, \beta_{1}\right) \neq l_{U},\left(\alpha_{2}, \beta_{2}\right) \neq l_{U}-$ собственные префиксы $(\alpha, \beta)$, причем $\left(\alpha_{2}, \beta_{2}\right)-$ префикс $\left(\alpha_{1}, \beta_{1}\right)$. Тогда $\tau(R)=\left(\alpha_{1}, \beta_{1}\right) R\left(\alpha_{2}, \beta_{2}\right)$.

В общем случае результат применения операции редукции следа к простому эксперименту $R$ означает «сжатие» эксперимента $R$ за счет удаления некоторых его внутренних частей.

Определение 2. Эксперимент $R^{\prime}$ назовем сокращенным (редуцируемым) $\tau$-подэкспериментом эксперимента $R$, если существует последовательность экспериментов $R=R_{1}, \ldots, R_{t}=R^{\prime}$ такая, что для любого $i \in\{2, \ldots, t\}$ эксперимент $R_{i}$ получен из эксперимента $R_{i-1}$ путем применения операции редукции эксперимента.

Для распространения операций редукции следа на кратные эксперименты рассмотрим эти эксперименты как следы о.-д. функций, реализуемых в заданных автоматах. Пусть $R-$ кратный эксперимент, содержащий простые эксперименты $R_{1}, \ldots, R_{t}(t \geq 2)$. Через $T(R)$ обозначим множество всех непустых подмножеств множества $\left\{R_{1}, \ldots, R_{t}\right\}$. Очевидно, что $R \in T(R)$, а также любой элемент $R \in T(R)$ есть кратный эксперимент, если $|R| \geq 2$ или простой, если $R=R_{i}(1 \leq i \leq t)$. Пусть $\left(\alpha_{1}, \beta_{1}\right),\left(\alpha_{2}, \beta_{2}\right)$ - префиксы всех вход-выходных слов (простых экспериментов, входящих в некоторый эксперимент $R \in T(R))$, причем $\left(\alpha_{1}, \beta_{1}\right)-$ префикс $\left(\alpha_{2}, \beta_{2}\right)$.

Определение 3. Полагаем, что эксперимент $R^{\prime}$ получен из эксперимента $R$ путем применения операции редукции следа: $R\left(\alpha_{1}, \beta_{1}\right) \rightarrow R\left(\alpha_{2}, \beta_{2}\right)$, если эксперимент $R^{\prime}$ может быть построен из эксперимента $R$ следующим образом: $R^{\prime}$ содержит все простые эксперименты $R$, не входящие в $R^{\prime}$, где $R \in T(R)$, а всякий простой эксперимент $R^{\prime \prime} \in R$ заменяется экспериментом $R$, полученным применением операции редукции следа: $R^{\prime \prime}\left(\alpha_{1}, \beta_{1}\right) \rightarrow R^{\prime \prime}\left(\alpha_{2}, \beta_{2}\right)$.

В общем случае операция редукции следа при распространении ее на кратные эксперименты является частичной. Кроме того, для кратных экспериментов операция редукции следа определяется тремя параметрами: $\left(\alpha_{1}, \beta_{1}\right),\left(\alpha_{2}, \beta_{2}\right)$ и экспериментом $R \in T(R)$. В зависимости от выбора этих параметров результат применения операции редукции следа к кратному эксперименту является простым или кратным экспериментом или же множеством вход-выходных слов, в котором существуют несовместимые вход-выходные слова.

Определение 4. Эксперимент $R^{\prime}$ назовем $\tau$-подэкспериментом эксперимента $R$, если существует последовательность множеств вход-выходных слов $R=R_{1}, \ldots, R_{t}=R^{\prime}$ такая, что для любого $i \in\{2, \ldots, t\}$ множество $R_{i}$ есть результат применения операции редукции следа к множеству $R_{i-1}$.

Определение 5. Множество экспериментов $W^{\prime}$ назовем $\tau$ подмножеством множества экспериментов $W$, если любой эксперимент $R^{\prime} \in W^{\prime}$ является $\tau$-подэкспериментом некоторого эксперимента $R \in B(W)$. Множество экспериментов $W^{\prime}-$ нетривиальное $\tau$-подмножество $W$, если существует $R^{\prime} \in W^{\prime}$, являющийся $\tau$-подэкспериментом эксперимента $R \in W$, отличный от $R$ и пустого слова.

Определение 6. След $W$ из $C(A)$ назовем $\tau$-неприводимым в $C(A)$, если любой его нетривиальный $\tau$-подэксперимент не принадлежит $C(A)$. 
Целью дальнейшего исследования будет изучение тупиковых $\tau$-неприводимых следов из $C(A)$, с использованием следов, идентифицирующих состояния неприводимых автоматов. При этом будут использоваться следующие понятия и результаты теории экспериментов с автоматами [4].

След $W$, идентифицирующий автомат $A$ из $K(U)$, идентифицирует состояние $s \in S_{A}$ автомата $A$ экспериментом $R_{s}$ множества $B(W)$ такой, что $R_{s} \subset \varphi_{s}(A)$ и для любого $s^{\prime} \neq s, s^{\prime} \in S_{A}, R_{s} \not \subset \varphi_{s}^{\prime}(A)$. Если $A-$ приведенный автомат с множеством состояний $S_{A}=\left\{s_{1}, \ldots, s_{n}\right\}$ и $W=\left\{R_{1}, \ldots, R_{n}\right\}-$ тупиковый след из $C(A)$, то для любых $i, j$ из $\{1, \ldots, n\}$ таких, что $i \neq j$ в множестве $B(W)$ существуют эксперименты $R_{i}$ и $R_{j}$, идентифицирующие состояния $s_{i}$ и $s_{j}$ соответственно, а следовательно, не совместимы между собой. Кроме того, для любых $i \in\{1, \ldots, n\}, x \in X$ и некоторого $y \in Y$ существует $i \in\{1, \ldots, n\}$ (зависящее от $x$ и $i$ ) такое, что $R_{i}(x, y)$ есть идентификатор состояния $s_{j}$, а следовательно, не совместимый с экспериментами $R=R_{1}, \ldots, R_{j-1}, R_{j+1}, \ldots, R_{n}$.

Рассмотрим некоторые, наиболее важные, частные случаи следов, идентифицирующих состояния неприводимых автоматов. Слово $\alpha \in X^{*}$ назовем диагностическим [4] для автомата $A=(S, X, Y, \delta, \lambda)$ с множеством состояний $s=\left\{s_{1}, \ldots, s_{n}\right\}$, если для любых $i, j$ из $\{1, \ldots, n\}$ таких, что $i \neq j$ следует, $\lambda\left(S_{i}, \alpha\right)=\lambda\left(S_{j}, \alpha\right)$. При этом множество простых экспериментов $\left(\alpha, \lambda\left(s_{1}, \alpha\right), \ldots, \alpha, \lambda\left(s_{n}, \alpha\right)\right)$, допустимых в автомате $A$ состояниями $s_{1}, \ldots, s_{n}$ соответственно, состоит из попарно несовместимых экспериментов, идентифицирующих состояния $s_{1}, \ldots, s_{n}$ соответственно. В [4] предложен метод построения и способ представления всех минимальных диагностических слов (если они есть) по заданному автомату $A$, используя понятия дерева преемников и диагностического дерева $Z(A)$. Пусть $\alpha \in X^{*}$ - слово, которому соответствует путь в $Z(A)$ [4] из начального состояния в финальное. Применяя операцию $\tau$, можно всегда получить $\tau$-подслово слова $\alpha$, соответствующее простому пути, ведущему в то же финальное состояние. Следовательно, для любого диагностического слова $\alpha \in X^{*}$ существует $\tau$-подслово $\alpha^{\prime}$, являющееся минимальным диагностическим словом. В то же время любое $\tau$-подслово минимального диагностического слова, отличного от него, не является диагностическим словом, хотя может и являться простым начальным идентификатором. Следовательно, множество всех $\tau$-неприводимых диагностических слов совпадает с множеством минимальных диагностических слов заданного автомата и конечно. Слово $\alpha \in X^{*}$ назовем контрольным для состояния $s_{i} \in S_{A}[4]$, если для любого $j \in\{1, \ldots, n\}$ из $\lambda\left(S_{i}, \alpha\right)=\lambda\left(S_{j}, \alpha\right)$ следует $s_{i}=s_{j}$. Если $\alpha \in X^{*}-$ контрольное слово состояния $s_{i}$, то эксперимент $\left(\alpha, \lambda\left(S_{i}, \alpha\right)\right)$ - начальный идентификатор состояния $s_{i}$. В [4] приведена верхняя оценка длины контрольного слова, равная $l=(\varphi / 12) n^{2}$, где $n=\left|S_{A}\right|$. Если в источнике $Z(A)$ в качестве финальных выбрать состояния, соответствующие $M$-группам, содержащим хотя бы одно простое $\Sigma$-множество, то $Z(A)$ будет представлять множество всех контрольных слов. Аналогично, множество всех $\tau$-неприводимых контрольных слов конечно в силу конечности множества всех простых путей в $Z(A)$, ведущих в конечное множество финальных состояний.

В то же время существуют автоматы, не имеющие ни диагностических, ни контрольных слов. В этом случае в таких автоматах для всякого состояния существуют кратные эксперименты [4], которые состоят из слов, представимых в $Z(A)$ при соответствующем выборе финальных состояний. Слово $\alpha \in X^{*}$ назовем различающим состояние $s \in S_{A}$ в автомате $A$, если существует непустое подмножество состояний $s_{i 1}, \ldots, s_{i r}$ автомата $A(r \leq n-1)$, не содержащее $s$ такое, что для любого $j \in\{1, \ldots, n\} \quad \lambda\left(S_{i j}, \alpha\right) \neq \lambda(S, \alpha)$. Множество состояний $s_{i 1}, \ldots, s_{i r}$, указанное в приведенном определении, назовем различимым множеством состояния $s$ по слову $\alpha$. Различающее слово $\alpha \in X^{*}$ состояния $s$ в $A$ назовем максимальным, если для любого $\alpha^{\prime} \in X^{*}$ такого, что $\alpha-$ префикс $\alpha^{\prime}$, различимые множества состояния $s$ по словам $\alpha$ и $\alpha^{\prime}$ совпадают.

Определение 7. Максимально различающее слово $\alpha \in X^{*}$ состояния $s$ назовем $\tau$-неприводимьм, если любое его $\tau$-подслово, отличное от $\alpha$, не является максимально различающим для состояния $s$.

Очевидно, что множество $\tau$-неприводимых максимально различающих слов состояния $s_{i} \in S_{A}(1 \leq i \leq n)$ конечно, так как им соответствует в источнике $Z(A)$ конечное множество простых (не проходящих через циклы диаграммы переходов) путей. Максимальная длина таких слов не превосходит оценки длины минимального диагностического слова.

Теорема. Для всякого приведенного автомата $A$ из $K(U)$ множество неприводимых следов, идентифицирующих автомат $A$, конечно.

Доказательство. Для всякого состояния любого приведенного автомата множество всех $\tau$-неприводимых максимально различающих слов конечно. Следовательно, любой элемент $\tau$-неприводимого тупикового эксперимента автомата может быть построен лишь конечным числом способов, так как 
он содержит конечное подмножество экспериментов, соответствующих $\tau$-неприводимым максимально различающим словам. Так как число таких элементов в $\tau$-неприводимом тупиковом эксперименте из $C(A)$ конечно, то и всех идентифицирующих следов для заданного автомата $A$ также конечно.

Таким образом, если в качестве «не избыточных» следов из $C(A)$ рассматривать $\tau$-неприводимые тупиковые следы из $C(A)$, то в $C(A)$ существует конечное множество таких следов.

\title{
Библиографический список
}

1. Льюинг Л. Идентификация систем. Теория для пользователя : пер. с англ. М. : Наука, 1991. 432 с.

2. Богомолов C. А. О синтезе автоматов по конечному множеству экспериментов // ДАН СССР. 1985. Т. 281, № 1. C. $20-22$.

3. Богомолов C. А. О восстановлении автомата по экспериментам // Дискретная математика. 1989. Т. 1, № 1. C. $135-146$.
4. Гилл А. Введение в теорию конечных автоматов. М. Наука, 1966. 272 с.

5. Кудрявиев В. Б., Алешин С. В., Подколзин А. С. Элементы теории автоматов. М. : Изд-во Моск. ун-та, 1978. $216 \mathrm{c}$.

6. Мур Э. Ф. Умозрительные эксперименты с последовательностными машинами // Автоматы / под ред. К. Э. Шеннона, Дж. Маккарти. М. : Изд-во иностр. лит., 1956. С. 179-210.

\section{Identification of a State Machine Structure with Finites Fragment of Behavior}

\begin{abstract}
S. A. Bogomolov
Saratov State Socio-Economic University, Russia, 410003, Saratov, Radishcheva st., 89, Alexbogomolov@ya.ru

Identification of a state machine structure with finite fragments of behavior is discussed. The state machine behavior is a set of various finite-sequential (f.-s.) functions realized in a state machine, and under a finite fragment of behavior we mean traces of f.-s. functions and state machines. The concept of an identifying trace for a state machine irredundant over its realization is introduced. The approach is suggested that enables to separate and descript in the set of traces identifying a state machine the finite set of irredundant traces consisting of only essential information for identification of a state machine.
\end{abstract}

Key words: state machine, experiments with a state machine, subexperiment of experiment, trace of f.-s. function and state machine, identifying trace of state machine, operation of trace reduction, irredundant identifying trace of state machine.

\section{References}

1. Ljung L. System Identification: Theory for the User. University of Linkoping Sweden, 1987. 432 p.

2. Bogomolov S. A. On the synthesis of automata from a finite set experiments. Dokl. Acad. Sci. USSR, 1985, vol. 281, no. 1, pp. 20-22.

3. Bogomolov S. A. Reconstruction of an automaton from experiments. Discrete Mathematics and Applications, 1991, vol. 1, no 2, pp. 117-128.
4. Gill A. Introduction to the Theory of Finite-state Machines. McGraw-Hill, 1962. 272 pp.

5. Kudryavtsev V. B., Aleshin S. V., Podkolzin A. S. Elementy teorii avtomatov [Topics of Automata Theory]. Moscow, Moscow Univ. Press, 1978. 216 pp. (in Russian). 6. Moor E. F. Speculative experiments with sequential machines. In Automata Studies, eds. C. E. Shennon, J. McCarthy. Princeton, Princeton Univ. Press, 1956, pp. 129-153.

УДК 519.872

\section{СЕТИ МАССОВОГО ОБСЛУЖИВАНИЯ С ГРУППОВЫМИ ПЕРЕХОДАМИ ТРЕБОВАНИЙ, БЛОКИРОВКАМИ И КЛАСТЕРАМИ}

\section{Ю. И. Митросранов ${ }^{1}$, В. И. Долгов ${ }^{2}$, Е. С. Рогачко ${ }^{3}$, Е. П. Станкевич ${ }^{4}$}

\footnotetext{
${ }^{1}$ Доктор технических наук, заведующий каседрой системного анализа и автоматического управления, Саратовский государственный университет им. Н. Г. Чернышевского, MitrophanovYul @info.sgu.ru ${ }^{2}$ Кандидат физико-математических наук, доцент кафредры системного анализа и автоматического управления, Саратовский государственный университет им. Н. Г. Чернышевского, www@ vidolgov.ru

${ }^{3}$ Кандидат фризико-математических наук, доцент кафредры системного анализа и автоматического управления, Саратовский государственный университет им. Н. Г. Чернышевского, RogachkoES@info.sgu.ru

${ }^{4}$ Старший преподаватель касредры системного анализа и автоматического управления, Саратовский государственный университет им. Н. Г. Чернышевского, StankevichElena@mail.ru
} 